\title{
Real-Time Molecular Scale Imaging of Dynamic Network Switching between Covalent Organic Frameworks
}

\author{
Gaolei Zhan, ${ }^{1 \ddagger}$ Zhen-Feng Cai, ${ }^{1 \neq *}$ Marta Martínez-Abadía, ${ }^{2}$ Aurelio Mateo-Alonso ${ }^{2,3}$ and Steven De \\ Feyter $^{1 *}$ \\ ${ }^{1}$ Department of Chemistry, Division of Molecular Imaging and Photonics, KU Leuven, Celestijnenlaan 200F, B-3001 Leuven, Belgium \\ ${ }^{2}$ POLYMAT, University of the Basque Country UPV/EHU, Avenida de Tolosa 72, Donostia-San Sebastian E-20018, Spain \\ ${ }^{3}$ Ikerbasque, Basque Foundation for Science, Bilbao, Spain.
}

Supporting Information

\begin{abstract}
The in situ on-surface conversion process from boroxine-linked covalent organic frameworks (COFs) to boronate ester-linked COFs is triggered and catalyzed, at room temperature, by an electric field and monitored with scanning tunneling microscopy (STM). The adaptive behavior within the generated dynamic covalent libraries (DCLs) was revealed, providing indepth understanding of the dynamic network switching process.
\end{abstract}

Covalent organic frameworks (COFs) are an emerging class of crystalline porous polymers formed by linking organic subunits into periodic two- and three-dimensional networks endowed with tailorable catalytic, optoelectronic, and molecular transport properties. ${ }^{1}$ The synthesis of such framework materials is based on reticular chemistry and the use of dynamic covalent chemistry (DCC) of various reaction types such as imine, ${ }^{2}$ boroxine, ${ }^{3}$ boronate ester, ${ }^{4}$ hydrazone, ${ }^{5}$ azine ${ }^{6}$ and imide. ${ }^{7}$ The dynamic nature of DCC provides for error-correction which favors the formation of crystalline products, and also allows for the shift of the dynamic system from one constitutional state to another one in response to external stimuli (e.g. light, ${ }^{8}$ temperature, ${ }^{9}$ pressure,${ }^{10}$ metal ions, ${ }^{11}$ protons, ${ }^{12}$ etc.). Following these principles, new synthetic strategies complementary to de novo synthesis have been developed very recently, targeting the post-modification of initially formed crystalline COFs or amorphous covalent organic polymers (COPs), to generate new COFs, known as COF-to-COF or COPto-COF transformation. ${ }^{13}$ Despite of its great significance in advancing synthetic methodology, little is known about the mechanistic aspects of such structural transformation process.

A potentially promising approach is to reduce the dimensionality of the problem, by confining the dynamic network switching to single-layered COFs (sCOFs) on a surface. DCC-based reactions (boroxine, boronate ester, imine) have indeed been successful in generating sCOFs on various surfaces..$^{3-4,14}$ Of particular interest are multi-component systems where building block exchange or linkage replacement is possible, potentially leading to network switching, COF-to-COF transformation being a special case. While the structure of sCOFs was characterized by scanning tunneling microscopy (STM) owing to its near-atomic resolution imaging ability, the dynamics of sCOFs formation processes remain hidden so far. The main challenge arises from the solvothermal reaction conditions requiring temperatures typically above $100{ }^{\circ} \mathrm{C}$, making it very difficult to follow the reaction dynamics with reasonable spatial and temporal resolution. In order to resolve these issues experimentally, two conditions are required: 1) in-situ, i.e. at the liquid-solid interface, continuous monitoring, ideally starting from the initiation of the reaction; 2) mild reaction conditions, i.e. at room-temperature. Both requirements are not compatible with solvothermal approaches.

Here we offer a unique approach to overcome these challenges, and a platform to investigate the dynamics of sCOFs synthesis and COF-to-COF transformation. Continuous monitoring and defining the start of the reaction: The large $\left(\mathrm{ca} .10^{9} \mathrm{~V} \mathrm{~m}^{-1}\right)$, highly-localized electric field generated between the STM tip and the surface has the potential to affect the kinetics and/or thermodynamics of chemical reactions, in line with theoretical and experimental work, proving that an oriented external electric field (EEF) can facilitate bond formation and bond cleavage reactions. ${ }^{15,16}$ Indeed, our recent study showed that by taking advantage of such large EEF, one can on-demand trigger the DCC-based polymerization and depolymerization processes of a monocomponent sCOFs, resulting in the reversible conversion between self-assembled molecular networks (SAMNs) of the monomer and SCOFs at a liquid/solid interface. ${ }^{17}$ Mild reaction conditions: By applying EEF, high temperatures were no longer required. The reactions now occur at room temperature, effectively facilitating the in-situ monitoring by STM.

Following the strategy outlined above, in this Communication, we chose the mixture of boronic acid and catechol compounds which gave rise to dynamic covalent libraries (DCLs) of three constituents bearing homogeneous linkages (boroxine or ester) or boroxineester heterogeneous linkages. By controlling the bias voltage between the STM tip and the surface, dynamic network switching from boroxine-linked COFs to boronate ester-linked COFs could be achieved (Figure 1). We also monitor the constitutional variation processes within the generated DCLs, and gain insight into the kinetics and thermodynamics ${ }^{18}$ as well as the $2 \mathrm{D}$ crystallization process, by using STM as a visualization tool and an external agent. 
The electric-field-mediated process described here facilitates the connection between different types of linkages and provides direct insights into the dynamic network switching process.

Two building blocks with $C_{3}$-symmetry (Figure 1, left panel) were used for this study carrying three boronic acid groups and three catechol groups, respectively: 1,3,5-tris(4-biphenylboronic acid) benzene (TBPBA) ${ }^{19}$, and 2,3,6,7,10,11-hexahydroxytriphenylene (HHTP).
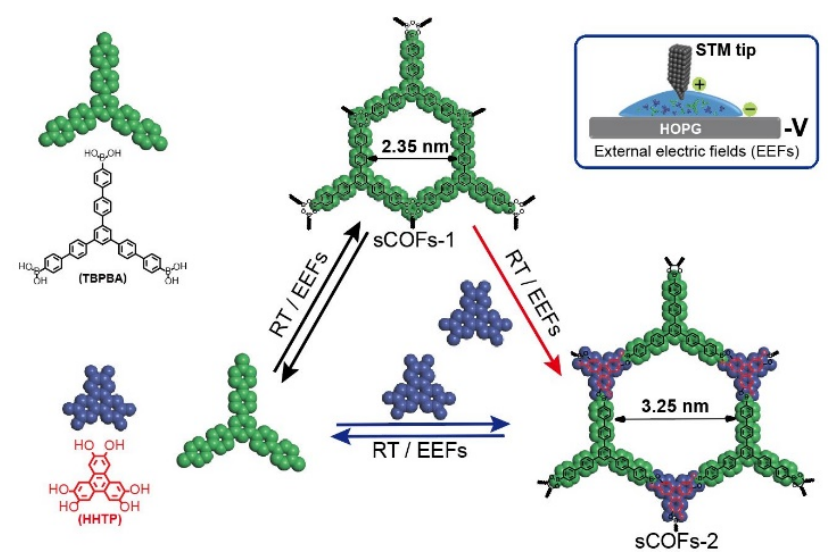

Figure 1. Chemical structures of TBPBA and HHTP (left) and schematic illustration (right) of external electric field (EEF) mediated network switching among self-assembled molecular networks (SAMNs), sCOFs-1 and sCOFs-2.

We start from the room-temperature deposition of about $10 \mu \mathrm{L}$ mixed solution $\left(5 \times 10^{-5} \mathrm{M}\right)$ of HHTP:TBPBA: $(1: 1 \mathrm{v} / \mathrm{v})$ in 1octanoic acid on a freshly cleaved highly oriented pyrolytic graphite (HOPG) surface. At positive sample bias (+ $0.3 \mathrm{~V})$, ordered close-packed domains and randomly distributed bright spots (blue arrow) with different sizes are observed on the surface (Figure 2a). High-resolution imaging (Figure 2c) shows the details of the close-packed phase, which is ascribed to the SAMNs of TBPBA molecules based on the measured size $(2.1 \pm 0.2 \mathrm{~nm})$ of each triangle feature. The bright spots are referred to as HHTP molecules since such features cannot be observed in absence of HHTP (Figure S1). Varying the solution stoichiometry (HHTPTBPBA) from 1:1 to 1:2 leads to reduced coverage of bright spots on the surface, and 2D co-assembly by the two components is never observed (Figure S2).

Switching the polarity of sample bias from positive to negative leads to a dynamic structural transition and by continuously scanning the same area, a regular porous network is eventually formed. Structural analysis of the calibrated STM images (Figure 2b,d) shows a hexagonal network with a unit cell vector of $3.1 \pm$ $0.1 \mathrm{~nm}$, which is in excellent agreement with the theoretical calculated size $(3.25 \mathrm{~nm})$ of the boronate ester-based 2D polymers (sCOFs-2). Submolecular resolution afforded by STM enables a clear identification of the triangular-shaped features of TPBPA and HHTP (Figure 2d). Linear or bended features inside the cavities of sCOFs-2 indicate the co-adsorption of solvent molecules of 1octanoic acid (Figure S3), which is not detected by scanning at positive sample bias. Figure $2 \mathrm{~d}$ also shows the proposed structural model of sCOFs-2. The covalent bond formation is supported by the same unit cell parameters of sCOFs-2 obtained with ex-situ synthesis approaches (Figure S4). The above results clearly demonstrate that, on-surface construction of sCOFs based on esterification can be achieved at room temperature, by controlling the polarity of sample bias.

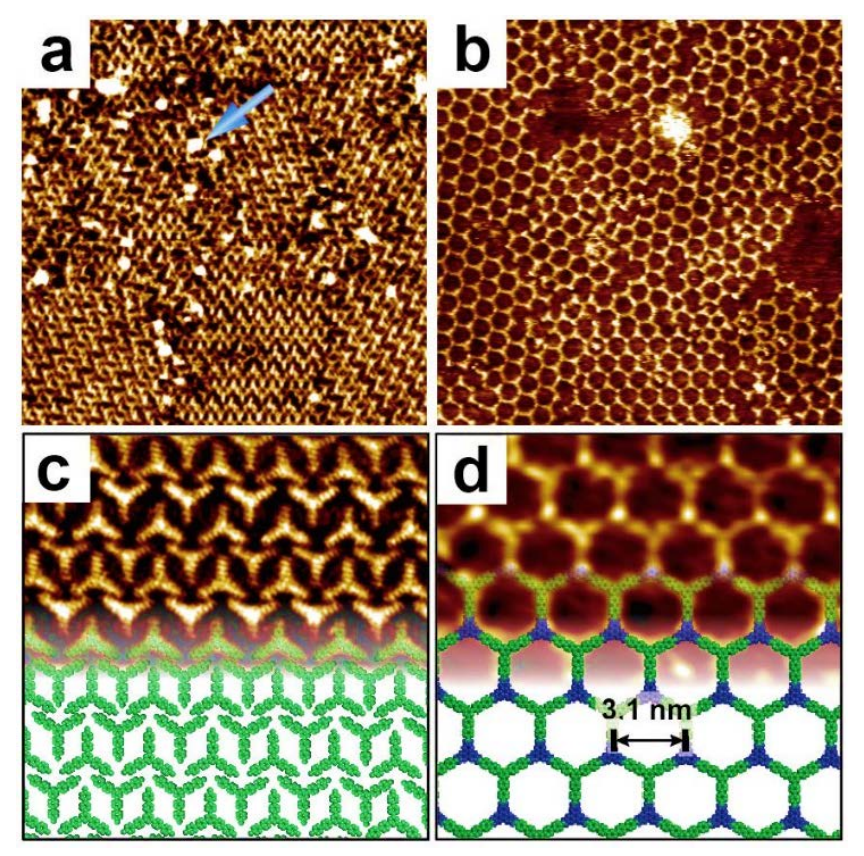

Figure 2. $(a-b)$ Large-scale and (c - d) high-resolution STM images of self-assembled TBPBA phase and boronate ester-based sCOFs-2, superimposed with proposed molecular models. Imaging conditions: (a, b) Scan size $=60 \times 60 \mathrm{~nm}^{2}, I_{\text {set }}=200 \mathrm{pA}, V_{\text {bias }}=0.3 \mathrm{~V}$; (c, d) Scan size $=18 \times$ $18 \mathrm{~nm}^{2}, I_{\text {set }}=200 \mathrm{pA}, V_{\text {bias }}=-0.6 \mathrm{~V}$.

The structural conversion process is time-dependent and is followed in situ, thus allowing for the real-time tracking of the pathways followed by sCOFs-2 formation. Immediately after reversing the bias, the transformation from SAMNs to boroxinebased sCOFs-1 takes place, resulting in a gradually increased coverage of sCOFs- 1 over time (Figure S5). After about 25 minutes, the coverage of sCOFs- 1 reaches a peak value $(\sim 45 \%)$, and at this time several islands of sCOFs-2 commence to appear (coverage $\sim 2 \%$ ) within the scanning region (Figure 3a). One can clearly distinguish one type of porous structure from another, thanks to the size differences between sCOFs-1 (2.3 nm) and sCOFs-2 (3.1 nm). Repeated scan cycles lead to the continuously increased coverage of sCOFs-2 and decreased coverage of sCOFs-1 (Figure 3 a-e, f). After c.a. 70 minutes, the surface is covered by sCOFs-2 with a coverage of around $40 \%$ (Figure $3 \mathrm{f}$ ) and only $\sim 3 \%$ of sCOFs- 1 remains. A detailed statistical analysis is provided in Figure S6. In contrast to the previously TBPBA mono-component system where a full coverage of sCOFs- 1 could be achieved and stabilized over time, ${ }^{17}$ the presence of HHTP greatly affects the process as every TPBPA molecule contains three boronic acid groups and each of them has the possibility to form either boroxine or boronate ester bonds. In addition to sCOFs- 1 and sCOFs-2 bearing the same type of linkages (boroxine or boronate ester), intermediates of boroxineester heterolinkages can also be distinguished (Figure S7). Due to the fact that all the DCL members share one common building block, i.e., TPBPA, scrambling occurs all over the experiment. On the one hand, the consumption of free boronic acid groups via esterification hinders the growth of sCOFs-1, which is supported by the fact that sCOFs- 1 domains of limited size are obtained 
(diameter of each domain $\mathbf{d}_{\mathbf{1}}$ was observed to be less than $10 \mathrm{~nm}$ ). On the other hand, the formation of sCOFs-2 is also influenced by boroxine formation since the growth of sCOFs-2 relies on the addition of HHTP species and boronate sources, which exist as either monomeric state or DCLs of various types.
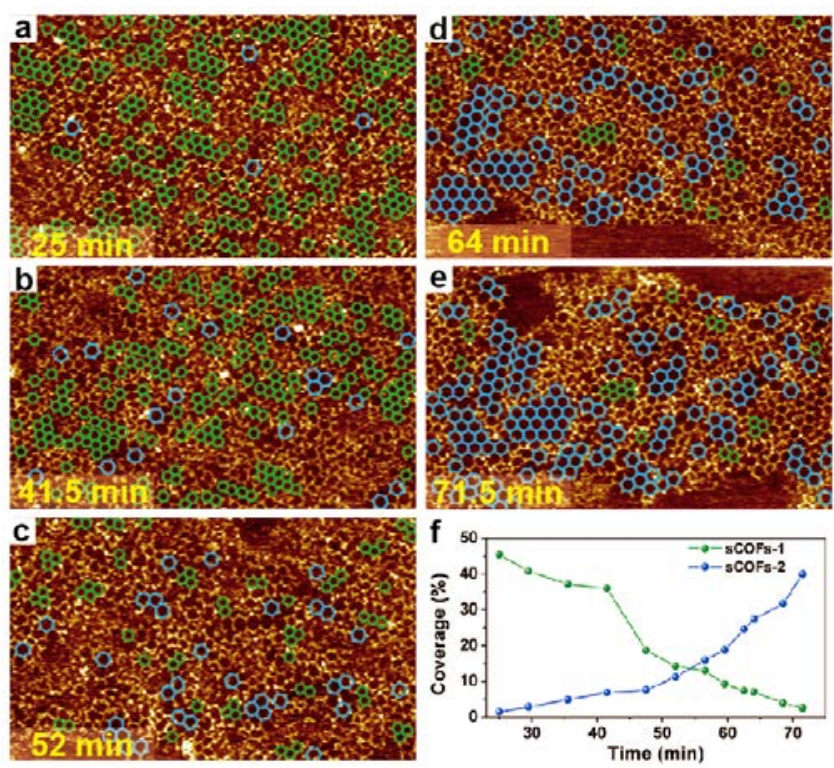

Figure 3. (a - e) Sequential STM images showing the time-dependent conversion process, from boroxine based sCOFs-1 towards boronate ester based sCOFs-2. For clarity, green and blue hexagons highlight the porous structures of sCOFs-1 and sCOFs-2, respectively. (f) Plot of the coverage of sCOFs- 1 and sCOFs- 2 against time. Imaging conditions: Scan size $=100$ $\times 60 \mathrm{~nm}^{2}, I_{\text {set }}=200 \mathrm{pA}, V_{\text {bias }}=-0.3 \mathrm{~V}$.

To understand the above-mentioned network switching process, from the initial distribution of mixed DCLs components to the selection/amplification of only one member of the library, we consider the following aspects. Firstly, there is a thermodynamic difference between boroxine and boronic ester formation, which has previously been followed with $\mathrm{H}^{1}$ NMR spectroscopy by employing different boronate sources. ${ }^{20}$ Boroxine is the kinetic product while boronate ester is the thermodynamic one, and esterification appears to be limited by hydrolysis of boroxine. These statements are in line with our in situ observation, that boronic acid monomers rapidly self-condensate to form boroxinebased products; and the increased coverage of sCOFs- 2 is accompanied by the reduced coverage or quasi-extinction of sCOFs-1 as a function of time (Figure 3f). A closer view of this process is shown in Figure 4, where one can clearly observe the nucleation and growth of sCOFs-2 at the expense of surrounding oligomers and sCOFs- 1 . To further validate that sCOFs- 2 can be obtained based on the COFs-to-COFs transformation strategy, we first generated sCOFs- 1 via an ex situ synthesis approach and then added HHTP. The results (Figure S8-9) show that sCOFs-2 can indeed be formed at room temperature, locally, after long-term continuous STM scanning, highlighting the importance of EEF. Secondly, the stabilization of crystalline products (sCOFs- 1 and sCOFs-2) is size-dependent. Compared to larger clusters, those with smaller sizes are more likely to undergo shrinkage or redissolution rather than elongation. In presence of HHTP, the creation of sCOFs-1 becomes size-limited $\left(\mathbf{d}_{\mathbf{1}}<10 \mathrm{~nm}\right)$ owing to the competitive reaction behavior. Therefore, the hydrolysis of sCOFs-1 is favored due to the lower stability of crystals of reduced size.

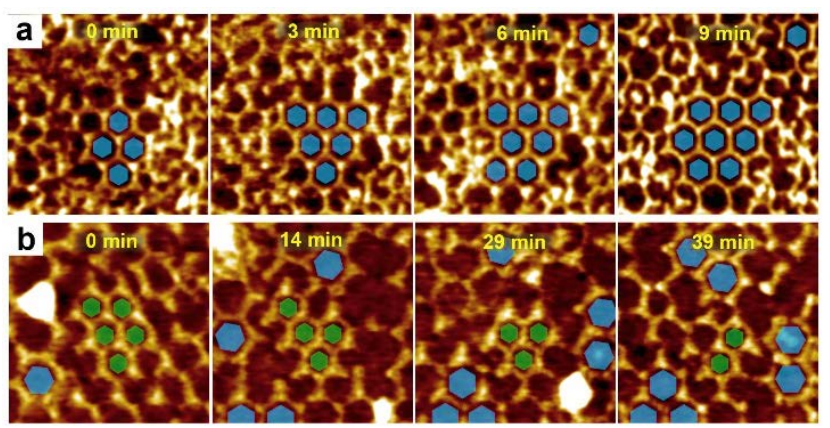

Figure 4. Sequential STM images showing the nucleation and growth of sCOFs-2 at the expense of surrounding oligomers and sCOFs-1. For clarity, green and blue hexagons highlight the porous structures of sCOFs-1 and sCOFs-2, respectively. Imaging conditions: (a) Scan size $=20 \times 20 \mathrm{~nm}^{2}, I_{\text {set }}$ $=150 \mathrm{pA}, V_{\text {bias }}=-0.5 \mathrm{~V}$; (b) Scan size $=15 \times 15 \mathrm{~nm}^{2}, I_{\text {set }}=200 \mathrm{pA}, V_{\text {bias }}=$ $-0.6 \mathrm{~V}$.

It is noteworthy that the conversion process from SAMNs to DCLs components is observed only when negative sample bias is applied. When the sample bias is reversed back to positive after the formation of DCLs components, self-assembled TBPBA phase can again be obtained on the surface (Figure S10). Such DCLs to SAMNs conversion process is induced locally and selectively, depending on the position and scanning area of the STM tip (Figure S11). The network switching between DCLs and SAMNs indicates the reversibility of the on-surface polymerization/depolymerization, which can be realized within the sample bias range of +0.2 to +0.7 $\mathrm{V}$ (depolymerization) and -0.2 to $-0.7 \mathrm{~V}$ (polymerization). The aforementioned phenomena, local reaction environment and reversible network switching (in response to the orientation of applied sample bias), support the statement that the EEF $\left(\sim 10^{9} \mathrm{~V} / \mathrm{m}\right)$ that exists between STM tip and the substrate triggers and catalyzes the reaction progress, and is a convenient approach to follow reaction dynamics at the nanoscale, notwithstanding the E-field effect is not fully understood yet.

In conclusion, we used 'EEF-mediated polymerization' to generate DCLs on HOPG surface based on dynamic boroxine and boronate ester formation. Time-dependent COF (boroxine) to COF (ester) conversion process was monitored with submolecular resolution by using in situ STM. The initialization of the on-surface polymerization/depolymerization process is realized, simply by controlling the applied sample bias. The direct visualization of the evolution routes provides for an in-depth understanding of the interplay between two types of COFs, on account of the difference in thermodynamic stabilities within DCLs and size-dependent stabilities of crystalline components. Our findings provide support for synthesizing boronate ester-based COFs starting from boroxine-based COFs via linkage conversion. This approach contributes to the construction methodology of 2D polymers, which may provide the foundation for the development of the next generation of dynamic covalent polymers for advanced materials applications. 


\section{ASSOCIATED CONTENT}

\section{Supporting Information}

The Supporting Information is available free of charge on the ACS Publications website at DOI: 10.1021/jacs.*******. Experimental methods and supporting figures (PDF)

\section{AUTHOR INFORMATION}

\author{
Corresponding Author \\ *zhenfeng.cai@kuleuven.be \\ steven.defeyter@kuleuven.be
}

\section{ORCID}

Gaolei Zhan: 0000-0002-7935-7998

Zhen-Feng Cai: 0000-0002-1659-4291

Marta Martínez-Abadía: 0000-0003-2068-0296

Aurelio Mateo-Alonso 0000-0002-5316-2594

Steven De Feyter: 0000-0002-0909-9292

\section{Author Contributions}

₹These authors contributed equally.

\section{Notes}

The authors declare no competing financial interests.

\section{ACKNOWLEDGMENT}

This work is supported by the Fund of Scientific Research-Flanders (FWO), in part by FWO under EOS 30489208, and KU Leuven Internal Grant 3E180504. We want to thank Prof. Kay Severin and Dr. E. Sheepwash for kindly providing the triboronic acids.

\section{REFERENCES}

(1) (a) Diercks, C. S.; Yaghi, O. M. The atom, the molecule, and the covalent organic framework. Science 2017, 355, 8. (b) Colson, J. W.; Woll, A. R.; Mukherjee, A.; Levendorf, M. P.; Spitler, E. L.; Shields, V. B.; Spencer, M. G.; Park, J.; Dichtel, W. R. Oriented 2D Covalent Organic Framework Thin Films on Single-Layer Graphene. Science 2011, 332, 228. (c) Côté, A. P.; Benin, A. I.; Ockwig, N. W.; O'Keeffe, M.; Matzger, A. J.; Yaghi, O. M. Porous, Crystalline, Covalent Organic Frameworks. Science 2005, 310, 1166. (d) Waller, P. J.; Gándara, F.; Yaghi, O. M. Chemistry of Covalent Organic Frameworks. Accounts of Chemical Research 2015, 48, 3053. (e) Dong, R.; Zhang, T.; Feng, $\mathrm{X}$. Interface-Assisted Synthesis of 2D Materials: Trend and Challenges. Chem. Rev. 2018, 118, 6189. (f) Sasmal, H. S.; Halder, A.; Kunjattu, S.; Dey, K.; Nadol, A.; Ajithkumar, T. G.; Bedadur, P. R.; Banerjee, R. Covalent Self-Assembly in Two Dimensions: Connecting Covalent Organic Framework Nanospheres into Crystalline and Porous Thin Films. J. Am. Chem. Soc. 2019, 141, 20371.

(2) (a) Xu, H.; Gao, J.; Jiang, D. Stable, crystalline, porous, covalent organic frameworks as a platform for chiral organocatalysts. Nat. Chem. 2015, 7, 905. (b) Belowich, M. E.; Stoddart, J. F. Dynamic imine chemistry. Chem. Soc. Rev. 2012, 41, 2003. (c) Lin, S.; Diercks, C. S.; Zhang, Y. B.; Kornienko, N.; Nichols, E. M.; Zhao, Y. B.; Paris, A. R.; Kim, D.; Yang, P.; Yaghi, O. M.; Chang, C. J. Covalent organic frameworks comprising cobalt porphyrins for catalytic CO2 reduction in water. Science 2015, 349, 1208. (d) Li, Y.; Chen, Q.; Xu, T.; Xie, Z.; Liu, J.; Yu, X.; Ma, S.; Qin, T.; Chen, L. De Novo Design and Facile Synthesis of 2D Covalent Organic Frameworks: A Two-in-One Strategy. $J$ Am Chem Soc 2019, 141, 13822. (e) Jakowetz, A. C.; Hinrichsen,
T. F.; Ascherl, L.; Sick, T.; Calik, M.; Auras, F.; Medina, D. D.; Friend, R. H.; Rao, A.; Bein, T. Excited-State Dynamics in Fully Conjugated 2D Covalent Organic Frameworks. J. Am. Chem. Soc. 2019, 141, 11565. (f) Ciesielski, A.; El Garah, M.; Haar, S.; Kovaříček, P.; Lehn, J.-M.; Samorì, P. Dynamic covalent chemistry of bisimines at the solid/liquid interface monitored by scanning tunnelling microscopy. Nat. Chem. 2014, 6, 1017.

(3) (a) Guan, C.-Z.; Wang, D.; Wan, L.-J. Construction and repair of highly ordered 2D covalent networks by chemical equilibrium regulation. Chem. Commun. 2012, 48, 2943. (b) Dienstmaier, J. F.; Medina, D. D.; Dogru, M.; Knochel, P.; Bein, T.; Heckl, W. M.; Lackinger, M. Isoreticular Two-Dimensional Covalent Organic Frameworks Synthesized by On-Surface Condensation of Diboronic Acids. ACS Nano 2012, 6, 7234. (c) Bilbao, N.; Martín, C.; Zhan, G.; Martínez-Abadía, M.; SanzMatías, A.; Mateo-Alonso, A.; Harvey, J. N.; Van der Auweraer, M.; Mali, K. S.; De Feyter, S. Anatomy of On-Surface Synthesized Boroxine Two-Dimensional Polymers. ACS Nano 2020, 14, 2354.

(4) (a) Yu, L.; Li, Z. B.; Wang, D. Construction of boronate ester based single-layered covalent organic frameworks. Chem. Commun. 2016, 52, 13771. (b) Liu, C.; Yu, Y.; Zhang, W.; Zeng, Q.; Lei, S. Room-Temperature Synthesis of Covalent Organic Frameworks with a Boronic Ester Linkage at the Liquid/Solid Interface. Chem.: Eur. J 2016, 22, 18412. (c) Martínez-Abadía, M.; Stoppiello, C. T.; Strutynski, K.; Lerma-Berlanga, B.; MartíGastaldo, C.; Saeki, A.; Melle-Franco, M.; Khlobystov, A. N.; Mateo-Alonso, A. A Wavy Two-Dimensional Covalent Organic Framework from Core-Twisted Polycyclic Aromatic Hydrocarbons. J. Am. Chem. Soc. 2019, 141, 14403.

(5) Stegbauer, L.; Schwinghammer, K.; Lotsch, B. V. A hydrazone-based covalent organic framework for photocatalytic hydrogen production. Chem. Sci. 2014, 5, 2789.

(6) (a) Kamiya, K.; Kamai, R.; Hashimoto, K.; Nakanishi, S. Platinum-modified covalent triazine frameworks hybridized with carbon nanoparticles as methanol-tolerant oxygen reduction electrocatalysts. Nat. Commun. 2014, 5, 5040. (b) Kuhn, P.; Antonietti, M.; Thomas, A. Porous, Covalent Triazine-Based Frameworks Prepared by Ionothermal Synthesis. Angew. Chem. Int. Edit. 2008, 47, 3450.

(7) Fang, Q.; Zhuang, Z.; Gu, S.; Kaspar, R. B.; Zheng, J.; Wang, J.; Qiu, S.; Yan, Y. Designed synthesis of large-pore crystalline polyimide covalent organic frameworks. Nat. Commun. 2014, 5, 4503.

(8) Ji, S.; Cao, W.; Yu, Y.; Xu, H. Dynamic Diselenide Bonds: Exchange Reaction Induced by Visible Light without Catalysis. Angew. Chem. Int. Edit. 2014, 53, 6781.

(9) (a) Giuseppone, N.; Lehn, J.-M. Protonic and Temperature Modulation of Constituent Expression by Component Selection in a Dynamic Combinatorial Library of Imines. Chem.: Eur. $J$ 2006, 12, 1715. (b) Herder, M.; Lehn, J.-M. The Photodynamic Covalent Bond: Sensitized Alkoxyamines as a Tool To Shift Reaction Networks Out-of-Equilibrium Using Light Energy. J. Am. Chem. Soc. 2018, 140, 7647.

(10) Sobczak, S.; Drożdż, W.; Lampronti, G. I.; Belenguer, A. M.; Katrusiak, A.; Stefankiewicz, A. R. Dynamic Covalent Chemistry under High-Pressure:A New Route to Disulfide Metathesis. Chem.: Eur. J 2018, 24, 8769.

(11) Hafezi, N.; Lehn, J.-M. Adaptation of Dynamic Covalent Systems of Imine Constituents to Medium Change by Component Redistribution under Reversible Phase Separation. J. Am. Chem. Soc. 2012, 134, 12861.

(12) Giuseppone, N.; Lehn, J.-M. Electric-Field Modulation of Component Exchange in Constitutional Dynamic Liquid Crystals. Angew. Chem. Int. Edit. 2006, 45, 4619.

(13) (a) Zhai, Y.; Liu, G.; Jin, F.; Zhang, Y.; Gong, X.; Miao, Z.; Li, J.; Zhang, M.; Cui, Y.; Zhang, L.; Liu, Y.; Zhang, H.; Zhao, Y.; Zeng, Y. Construction of Covalent-Organic Frameworks 
(COFs) from Amorphous Covalent Organic Polymers via Linkage Replacement. Angew. Chem., Int. Ed. 2019, 58, 17679. (b) Segura, J. L.; Royuela, S.; Mar Ramos, M. Post-synthetic modification of covalent organic frameworks. Chem Soc Rev 2019, 48, 3903. (c) Miao, Z.; Liu, G.; Cui, Y.; Liu, Z.; Li, J.; Han, F.; Liu, Y.; Sun, X.; Gong, X.; Zhai, Y.; Zhao, Y.; Zeng, Y. A Novel Strategy for the Construction of Covalent Organic Frameworks from Nonporous Covalent Organic Polymers. Angew. Chem., Int. Ed. 2019, 58, 4906. (d) Li, Z.; Ding, X.; Feng, Y.; Feng, W.; Han, B.-H. Structural and Dimensional Transformations between Covalent Organic Frameworks via Linker Exchange. Macromolecules 2019, 52, 1257. (e) Qian, C.; Qi, Q.-Y.; Jiang, G.-F.; Cui, F.-Z.; Tian, Y.; Zhao, X. Toward Covalent Organic Frameworks Bearing Three Different Kinds of Pores: The Strategy for Construction and COFto-COF Transformation via Heterogeneous Linker Exchange. $J$. Am. Chem. Soc. 2017, 139, 6736.

(14) (a) Liu, X.-H.; Guan, C.-Z.; Ding, S.-Y.; Wang, W.; Yan, H.-J.; Wang, D.; Wan, L.-J. On-Surface Synthesis of SingleLayered Two-Dimensional Covalent Organic Frameworks via Solid-Vapor Interface Reactions. J Am Chem Soc 2013, 135, 10470. (b) Clair, S.; de Oteyza, D. G. Controlling a Chemical Coupling Reaction on a Surface: Tools and Strategies for OnSurface Synthesis. Chem. Rev. 2019, 119, 4717. (c) Bilbao, N.; Yu, Y.; Verstraete, L.; Lin, J.; Lei, S.; De Feyter, S. The impact of grafted surface defects on the on-surface Schiff-base chemistry at the solid-liquid interface. Chem. Commun. 2018, 54, 9905.

(15) (a) Aragones, A. C.; Aworth, N. L. H.; Darwish, N.; Iampi, S. C.; Bloomfield, N. J.; Wallace, G. G.; Diez-Perez, I.;
Coote, M. L. Electrostatic catalysis of a Diels-Alder reaction. Nature 2016, 531, 88. (b) Meir, R.; Chen, H.; Lai, W.; Shaik, S. Oriented Electric Fields Accelerate Diels-Alder Reactions and Control the endo/exo Selectivity. ChemPhysChem 2010, 11, 301.

(16) Zhang, L.; Laborda, E.; Darwish, N.; Noble, B. B.; Tyrell, J. H.; Pluczyk, S.; Le Brun, A. P.; Wallace, G. G.; Gonzalez, J.; Coote, M. L.; Ciampi, S. Electrochemical and Electrostatic Cleavage of Alkoxyamines. J Am Chem Soc 2018, 140, 766.

(17) Cai, Z.-F.; Zhan, G.; Daukiya, L.; Eyley, S.; Thielemans, W.; Severin, K.; De Feyter, S. Electric-Field-Mediated Reversible Transformation between Supramolecular Networks and Covalent Organic Frameworks. J Am Chem Soc 2019, 141, 11404.

(18) Kathan, M.; Kovaříček, P.; Jurissek, C.; Senf, A.; Dallmann, A.; Thünemann, A. F.; Hecht, S. Control of Imine Exchange Kinetics with Photoswitches to Modulate Self-Healing in Polysiloxane Networks by Light Illumination. Angew. Chem. Int. Edit. 2016, 55, 13882.

(19) Sheepwash, E.; Krampl, V.; Scopelliti, R.; Sereda, O.; Neels, A.; Severin, K. Molecular Networks Based on Dative Boron-Nitrogen Bonds. Angew. Chem. Int. Edit. 2011, 50, 3034.

(20) Spitler, E. L.; Giovino, M. R.; White, S. L.; Dichtel, W. R. A mechanistic study of Lewis acid-catalyzed covalent organic framework formation. Chem Sci 2011, 2, 1588. 
TOC:

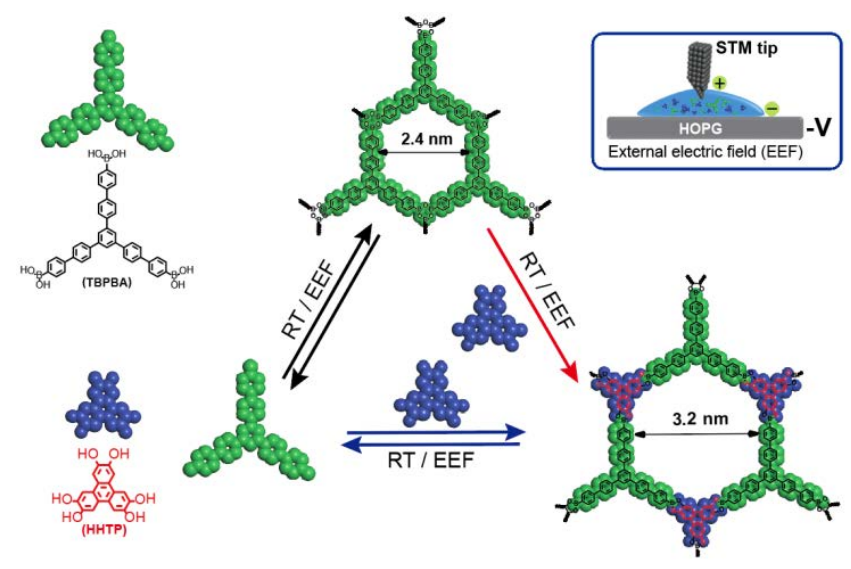

